\title{
A fast and easy-to-implement inversion algorithm for mobility particle size spectrometers considering particle number size distribution information outside of the detection range
}

\author{
S. Pfeifer ${ }^{1}$, W. Birmili ${ }^{1}$, A. Schladitz ${ }^{1,2}$, T. Müller ${ }^{1}$, A. Nowak ${ }^{1,3}$, and A. Wiedensohler ${ }^{1}$ \\ ${ }^{1}$ Leibniz Institute for Tropospheric Research, Permoserstraße 15, 04318 Leipzig, Germany \\ ${ }^{2}$ Saxon State Office for Environment, Agriculture and Geology, Pillnitzer Platz 3, 01326 Dresden, Germany \\ ${ }^{3}$ Physikalisch-Technische Bundesanstalt, Bundesallee 100, 38116 Braunschweig, Germany
}

Correspondence to: S. Pfeifer (pfeifer@ tropos.de)

Received: 19 April 2013 - Published in Atmos. Meas. Tech. Discuss.: 29 May 2013

Revised: 19 September 2013 - Accepted: 2 December 2013 - Published: 14 January 2014

\begin{abstract}
Multiple-charge inversion is an essential procedure to convert the raw mobility distributions recorded by mobility particle size spectrometers, such as the DMPS or SMPS (differential or scanning mobility particle sizers), into true particle number size distributions. In this work, we present a fast and easy-to-implement multiple-charge inversion algorithm with sufficient precision for atmospheric conditions, but extended functionality. The algorithm can incorporate size distribution information from sensors that measure beyond the upper sizing limit of the mobility spectrometer, such as an aerodynamic particle sizer (APS) or an optical particle counter (OPC). This feature can considerably improve the multiple-charge inversion result in the upper size range of the mobility spectrometer, for example, when substantial numbers of coarse particles are present. The program also yields a continuous size distribution from both sensors as an output. The algorithm is able to calculate the propagation of measurement errors, such as those based on counting statistics, into on the final particle number size distribution. As an additional aspect, the algorithm can perform all inversion steps under the assumption of non-spherical particle shape, including constant or size-dependent shape factors.
\end{abstract}

\section{Introduction}

Mobility particle size spectrometers are widely used in aerosol science and have enjoyed broad application in both laboratory and field studies (Knutson and Whitby, 1975; Kousaka et al., 1985; McMurry, 2000). Rather than a true particle number size distribution, they measure an electrical particle mobility distribution. Knowing the bipolar charge distribution (Wiedensohler, 1988) and the instrument responses of the differential mobility analyser (DMA) and the particle counter or condensation particle counter (CPC), it is possible to convey the electrical particle mobility distribution into the true particle number size distribution. This procedure has been called multiple-charge inversion (Alofs and Balakumar, 1982; Kandlikar and Ramachandran, 1999). Surprisingly few publications are available that specify their algorithm in detail or the occupation of the matrix (e.g. Brunner, 2007). Wiedensohler et al. (2012) highlighted the need to characterize the performance of multiple-charge inversion routines as part of an attempt to enhance the mutual comparability of worldwide atmospheric aerosol measurements. In their work, 12 contemporary multiple-charge inversion routines showed deviations of up to $5 \%$ with respect to the resulting particle number concentration. The deviations were attributed, among others things, to different physical constants and charging probabilities used, different solutions to the matrix inversion problem, and different approaches of how to discretize, pre-process and post-process the data.

Only few of such inversion routines have been designed to handle size distribution information from multiple sensors. Some of our previous work highlighted, for example, the need to complement sub-micrometre mobility spectrometer information by additional super-micrometre size distribution measurements in specific atmospheric situations, such as dust plumes (Birmili et al., 2008; Schladitz et al., 2009). A lack of super-micrometre size distribution information could 
then lead to a drastic overestimation of the particle number size distribution in the upper size range of the mobility spectrometer. In practice, the implementation of integrative inversion routines will closely depend on the type of available instrumental parameters. Fiebig et al. (2005), for example, proposed an iterative method that is able to merge information from multiple size-selective instruments involving a differential mobility analyser. Last but not least, it might be of additional benefit for an inversion routine to be compact and be able to easily process raw data during real-time data acquisition, as well as large amounts of data during post-processing.

In this work, we present the theoretical framework of a new inversion algorithm that inverts electrical particle mobility distribution from a mobility particle size spectrometer in conjunction with data from an aerodynamic particle sizer (APS) or an optical particle counter (OPC). The algorithm is designed for a maximum speed, while trying to remain as accurate as possible with a minimum of assumptions. The framework allows for handling of non-spherical particles, with particle shape being represented either by a constant shape factor or a size-dependent shape factor profile. A key feature of the algorithm is that we attempted to keep an analytical solution of the multiple-charge inversion problem, thus avoiding numerical iteration, which is possible at the expense of some accuracy as well as noise reduction. This is a distinction, for example, from other algorithms that apply external constraints such as non-negativity and smoothness (e.g. Talukdar and Swihart, 2003).

Due to the underlying strict system of linear equations, we are further able to compute how measurement errors, such as those based on counting statistics, propagate into the final particle number size distribution. The new capabilities of the algorithm are illustrated for two example cases.

\section{Theory}

The measured electrical particle mobility distribution (EPMD) $f^{*}$ can be written as a convolution integral of the real particle number size distribution (PNSD) $f$ and the transfer function $h$ with the electrical particle mobility $Z$ as independent variable. ${ }^{1}$

$f^{*}(Z)=(f * h)(Z)=\int_{-\infty}^{\infty} f\left(Z^{\prime}\right) h\left(Z-Z^{\prime}\right) \mathrm{d} Z^{\prime}$.

It is possible to calculate the PNSD from the measured EPMD by deconvolution. From the beginning, we abandon the attempt to find the direct analytic solution of the transfer function for a deconvolution. The measured EPMD is given at $N$ discrete mobility sampling points, so the problem is transformed to a system of equations which is easy to solve:

\footnotetext{
${ }^{1}$ A diameter $D$ scaled $x$ axis is also possible, but is not used in this work.
}

$$
\left(\begin{array}{c}
f_{1}^{*} \\
\vdots \\
f_{N}^{*}
\end{array}\right)=\left(\begin{array}{ccc}
a_{11} & \ldots & a_{1 N} \\
\vdots & \ddots & \vdots \\
a_{N 1} & \ldots & a_{N N}
\end{array}\right)\left(\begin{array}{c}
f_{1} \\
\vdots \\
f_{N}
\end{array}\right) .
$$

Since we intend to consider non spherical particles in the algorithm, we employ the volume-equivalent particle diameter $D_{\text {pve }}$ as the size parameter.

However, the DMA selects particles according to their electrical mobility

$Z=\frac{n q_{\mathrm{e}}}{3 \pi \eta} \frac{C_{\mathrm{c}}\left(D_{\mathrm{pm}}\right)}{D_{\mathrm{pm}}}$

where $D_{\mathrm{pm}}$ is the mobility diameter and $C_{\mathrm{C}}$ is the Cunningham correction factor. Often, the EPMD is already logged as particle mobility diameter for singly charged particles (Wiedensohler et al., 2012). These grid points can be recalculated to volume-equivalent diameter using the transformation formula

$\frac{D_{\mathrm{pm}}}{C_{\mathrm{c}}\left(D_{\mathrm{pm}}\right)}=\chi\left(D_{\mathrm{pve}}\right) \frac{D_{\mathrm{pve}}}{C_{\mathrm{c}}\left(D_{\mathrm{pve}}\right)}$,

where $\chi\left(D_{\text {pve }}\right)$ is the size-dependent aerodynamic shape factor, defined as the ratio of the drag of the arbitrarily shaped particle and the volume-equivalent sphere, in the Stokes regime. Examples of shape factors of real particles are provided, for example, in Hinds (1999, p. 52).

\subsection{DMA transfer function}

The transfer function $g\left(Z, Z^{\prime}\right)$ of particles through a DMA at the position $Z$ can be simply described by a triangular function with the height $\alpha(Z)$ and the dimensionless half-width $\beta(Z)$ or the dimensionless area $A(Z)=\alpha(Z) \beta(Z)$. The size dependency ensures a consideration of diffusional broadening of this transfer function (Birmili et al., 1997).

Ignoring multiply charged particles, as well as the counting efficiency of the CPC, the concentration downstream the DMA is given by the integral

$c(Z)=\int_{-\infty}^{\infty} \frac{\mathrm{d} c}{\mathrm{~d} Z^{\prime}}\left(Z^{\prime}\right) g\left(Z, Z^{\prime}\right) \mathrm{d} Z^{\prime}$,

while the transfer function (see Fig. 1) is given by Knutson and Whitby (1975):

$$
\begin{aligned}
& g\left(Z, Z^{\prime}\right)= \\
& \begin{cases}\frac{\alpha}{2 \beta}\left(\left|\frac{Z^{\prime}}{Z}-(1+\beta)\right|+\left|\frac{Z^{\prime}}{Z}-(1-\beta)\right|-2\left|\frac{Z^{\prime}}{Z}-1\right|\right) & Z^{\prime} \in((1-\beta) Z,(1+\beta) Z) \\
0 & \text { otherwise }\end{cases}
\end{aligned}
$$

In the case of an almost constant true PNSD, slowly varying with $Z^{\prime}$, in the range of $(1-\beta) Z$ and $(1+\beta) Z$, the convolution can be approximated (Stratmann et al., 1997) by 


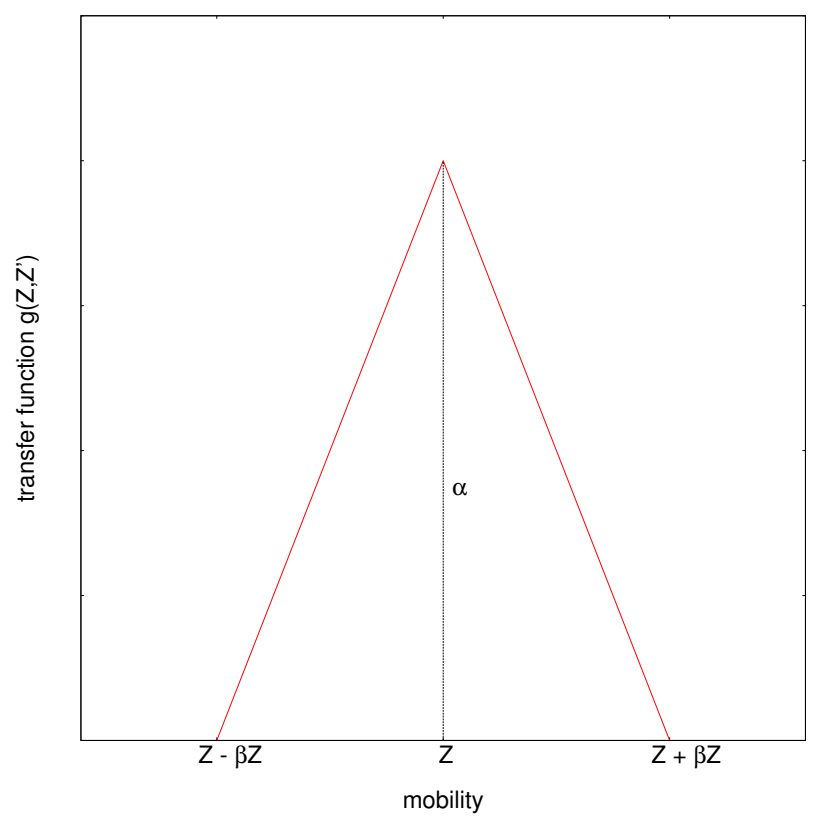

Fig. 1. Schematic of a triangular DMA transfer function

$c(Z) \simeq \mathrm{d} c / \mathrm{d} \ln Z(Z) A(Z)$. With a conversion factor $C_{i}$ (see Appendix A) we obtain the same approximation for the common unit $\mathrm{d} c / \mathrm{d} \log D$. Considering also the CPC counting efficiency $E_{C}$, we can find an expression for the DMA transfer function using this approximation:

$$
\begin{aligned}
& h_{\mathrm{dma}}\left(Z-Z^{\prime}\right)= \\
& \quad E_{C}\left(D_{\mathrm{pve}}(Z)\right) A(Z) C_{i}\left(D_{\mathrm{pve}}(Z)\right) \delta\left(Z^{\prime}-Z\right),
\end{aligned}
$$

where $\delta$ is the Dirac delta function. It should be mentioned that, under conditions of atmospheric aerosol particles, the approximation is adequate due to weak variations of the PNSD in the range of the transfer function. For narrow particle size distributions, such as those generated in the laboratory, this approximation is expected to be invalid. For such conditions, it is necessary to consider also the real shape of the transfer function.

\subsection{Charging probability and transfer function of multiply charged particles}

As already mentioned, we employ the volume-equivalent particle diameter $D_{\text {pve }}$ as the size parameter.

However, previous studies have shown that the bipolar diffusion charging for moderate non-spherical particles is similar to a sphere with the same mobility (Rogak and Flagan, 1992). Hence we use the mobility equivalent diameter $D_{\mathrm{pm}}$ as the size input to calculate the probability of multiply charged particles in a bipolar charge equilibrium. For strong non-spherical particles, for example particles with large aspect ratios, this approach seems to be invalid ( $\mathrm{Ku}$ et al., 2011).
Table 1. Fit parameter for specific charge of the fifth-degree polynomial approximation of Wiedensohler (1988).

\begin{tabular}{lrrrrr}
\hline & & \multicolumn{3}{c}{$N$} \\
$a_{i}(N)$ & -2 & -1 & 0 & +1 & +2 \\
\hline$a_{0}$ & -26.3328 & -2.3197 & -0.0003 & -2.3484 & -44.4756 \\
$a_{1}$ & 35.9044 & 0.6175 & -0.1014 & 0.6044 & 79.3772 \\
$a_{2}$ & -21.4608 & 0.6201 & 0.3073 & 0.4800 & 62.8900 \\
$a_{3}$ & 7.0867 & -0.1105 & -0.3372 & 0.0013 & 26.4492 \\
$a_{4}$ & -1.3088 & -0.1260 & 0.1023 & -0.1553 & -5.7480 \\
$a_{5}$ & 0.1051 & 0.0297 & -0.0105 & 0.0320 & 0.5059 \\
\hline
\end{tabular}

For singly and doubly charged particles smaller than $1 \mu \mathrm{m}$, we use the analytical approximation formula given by Wiedensohler (1988):

$p\left(D_{\mathrm{pm}}, n\right)=10^{\left[\sum_{i=0}^{5} a_{i}(n)\left(\log \frac{D_{\mathrm{pm}}}{\mathrm{nm}}\right)^{i}\right]}$,

where $a_{i}(n)$ are the fit parameters of the fifth-degree polynomial approximation (see Table 1).

For larger particles or more highly charged particles we use the charge probability distribution by Gunn (1955):

$$
\begin{aligned}
p\left(D_{\mathrm{pm}}, n\right) & =\frac{1}{\sqrt{2 \pi \sigma}} \exp \left[-\frac{(n-\sigma \ln (0.875))^{2}}{2 \sigma}\right] \\
\sigma & =\frac{2 \pi \epsilon_{0} D_{\mathrm{pm}} k T}{q_{e}{ }^{2}} .
\end{aligned}
$$

The measured EPMD is influenced by multiple charges; that is, for a given mobility diameter a mobility particle size spectrometer detects not only singly charged particles with the electrical mobility $Z$ but also larger, multiply charged particles with the same electrical mobility $Z / n$ ( $n=2,3, \ldots$ ), where $Z$ is the electrical mobility for singly charged particles, and also in the following sections.

Using Eqs. (8) and (9) we calculate the probability of specific multiple charges $p\left(D_{\mathrm{pve}}, n\right)$. The transfer function to consider multiple charges $h_{\text {cha }}$ is

$$
\begin{aligned}
h_{\text {cha }}\left(Z-Z^{\prime}\right) & =p\left(D_{\mathrm{pm}}\left(\frac{1}{1} Z\right), n=1\right) \delta\left(Z^{\prime}-\frac{1}{1} Z\right) \\
& +p\left(D_{\mathrm{pm}}\left(\frac{1}{2} Z\right), n=2\right) \delta\left(Z^{\prime}-\frac{1}{2} Z\right) \\
& +\ldots \\
& +p\left(D_{\mathrm{pm}}\left(\frac{1}{k} Z\right), n=k\right) \delta\left(Z^{\prime}-\frac{1}{k} Z\right) \\
& +\ldots \\
& =\sum_{n=1}^{\infty} p\left(D_{\mathrm{pm}}\left(\frac{1}{n} Z\right), n\right) \delta\left(Z^{\prime}-\frac{1}{n} Z\right) .
\end{aligned}
$$

\subsection{Resulting system of equations}

The total transfer function $h$ is the convolution of the DMA transfer function (Eq. 7) with the transfer function for 
Table 2. Symbol directory.

\begin{tabular}{|c|c|}
\hline Symbol & Explanation \\
\hline$D_{\text {pve }}$ & particle diameter (volume equivalent) \\
\hline$D_{\mathrm{pm}}$ & particle diameter (mobility equivalent) \\
\hline$n$ & number of charges \\
\hline$q_{\mathrm{e}}$ & elementary charge \\
\hline$\eta$ & dynamic viscosity \\
\hline$\chi\left(D_{\mathrm{pve}}\right)$ & size-dependent aerodynamic shape factor \\
\hline$Z\left(D_{\text {pve }}, n\right)$ & electrical particle mobility \\
\hline$\alpha$ & height of the DMA transfer function \\
\hline$\beta$ & $\begin{array}{l}\text { dimensionless width of DMA transfer func- } \\
\text { tion }\end{array}$ \\
\hline$A$ & $\begin{array}{l}\text { dimensionless area of DMA transfer func- } \\
\text { tion }\end{array}$ \\
\hline$f$ & $\begin{array}{l}\text { real particle number size distribution } \\
\text { (PNSD) }\end{array}$ \\
\hline$f^{*}$ & $\begin{array}{l}\text { measured electrical particle mobility distri- } \\
\text { bution (EPMD) }\end{array}$ \\
\hline$p\left(D_{\mathrm{p}}, n\right)$ & charge probability \\
\hline$\delta$ & Dirac delta function \\
\hline$h_{\mathrm{dma}}$ & $\begin{array}{l}\text { approximated DMA transfer function, con- } \\
\text { sidering the transmission }\end{array}$ \\
\hline$h_{\text {cha }}$ & $\begin{array}{l}\text { transfer function, considering the multiple } \\
\text { charges }\end{array}$ \\
\hline$h$ & total transfer function \\
\hline$C_{i}\left(D_{\mathrm{pve}}\right)$ & $\begin{array}{l}\text { conversion factor from } \mathrm{d} n / \mathrm{d} \ln Z \text { to } \\
\mathrm{d} n / \mathrm{d} \log D\end{array}$ \\
\hline $\mathbf{A}$ & multiple-charge matrix \\
\hline $\mathbf{L}$ & transformation matrix, inverse of $\mathbf{A}$ \\
\hline $\mathbf{L}_{\mathrm{var}}$ & transformation matrix of variance \\
\hline
\end{tabular}

multiply charged particles (Eq. 10):

$$
\begin{aligned}
& h\left(Z-Z^{\prime}\right)=\sum_{n=1}^{\infty} p\left(D_{\mathrm{pm}}\left(\frac{1}{n} Z\right), n\right) E_{C}\left(D_{\mathrm{pve}}\left(\frac{1}{n} Z\right)\right) \\
& A\left(\frac{1}{n} Z\right) C_{i}\left(D_{\mathrm{pve}}\left(\frac{1}{n} Z\right)\right) \delta\left(Z^{\prime}-\frac{1}{n} Z\right) .
\end{aligned}
$$

Let $E$ be the value of efficiency by combining the probability of multiply charged particles and the DMA efficiency inclusive the conversion factor with

$$
\begin{gathered}
E(Z, n)=p\left(D_{\mathrm{pve}}\left(\frac{1}{n} Z\right), n\right) E_{C}\left(D_{\mathrm{pve}}\left(\frac{1}{n} Z\right)\right) \\
A\left(\frac{1}{n} Z\right) C_{i}\left(D_{\mathrm{pve}}\left(\frac{1}{n} Z\right)\right) .
\end{gathered}
$$

As an example, in Appendix $\mathrm{C}$, one can see the simple modification of the efficiency $E$ in the case of using a cloud condensation nuclei counter (CCNC) instead of a CPC. Finally, we obtain an equation for the measured electrical particle mobility distribution at $Z$ as a function of the real PNSD:

$$
\begin{aligned}
f^{*}(Z) & =\int_{-\infty}^{\infty} f\left(Z^{\prime}\right) \sum_{n=1}^{\infty} E(Z, n) \delta\left(Z^{\prime}-\frac{1}{n} Z\right) \mathrm{d} Z^{\prime} \\
& =\sum_{n=1}^{\infty} E(Z, n) \int_{-\infty}^{\infty} f\left(Z^{\prime}\right) \delta\left(Z^{\prime}-\frac{1}{n} Z\right) \mathrm{d} Z^{\prime} \\
& =\sum_{n=1}^{\infty} E(Z, n) f\left(\frac{1}{n} Z\right) .
\end{aligned}
$$

As previously mentioned, the measured EPMD is given in $N$ discrete mobility sampling points, where

$$
f_{i}^{*}=f^{*}\left(Z_{i}\right)=\sum_{n=1}^{\infty} E\left(Z_{i}, n\right) f\left(\frac{1}{n} Z_{i}\right) .
$$

By using linear interpolation, given in Appendix B, we obtain

$$
\begin{gathered}
f\left(\frac{1}{n} Z_{i}\right)=\frac{\frac{1}{n} Z_{i}-Z_{j}}{Z_{j+1}-Z_{j}} f_{j+1}+\frac{Z_{j+1}-\frac{1}{n} Z_{i}}{Z_{j+1}-Z_{j}} f_{j} \\
\frac{1}{n} Z_{i} \in\left(Z_{j+1}, Z_{j}\right) .
\end{gathered}
$$

The sampling points of the real PNSD should be identical to those of the measured EPMD. With this approach we are as close as possible to the actual information content of the measurement without additional assumptions. Thus we found a system of equations for the multiply charge inversion with the entries of matrix $\mathbf{A}$ :

$a_{i j}=\sum_{n=1}^{\infty} E\left(Z_{i}, n\right)\left\{\begin{array}{ll}\frac{\frac{1}{n} Z_{i}-Z_{j-1}}{Z_{j}-Z_{j-1}} & \frac{1}{n} Z_{i} \in\left(Z_{j}, Z_{j-1}\right) \\ 1 & \frac{1}{n} Z_{i}=Z_{j} \\ \frac{Z_{j+1}-\frac{1}{n} Z_{i}}{Z_{j+1}-Z_{j}} & \frac{1}{n} Z_{i} \in\left(Z_{j+1}, Z_{j}\right) \\ 0 & \text { otherwise }\end{array}\right.$,

with

$f^{*}=\mathbf{A} \cdot f$.

Because the number and position of the sampling points of the inverted PNSD and the measured EPMD should be identical, the matrix $\mathbf{A}$ is quadratic. Furthermore, due to $i>j \Rightarrow a_{i j}=0$, it is an upper triangular matrix, or a less occupied upper triangular matrix, which is easy to solve with a unique solution, e.g. using a simple Gauss-Jordan algorithm. Let $\mathbf{L}$ be the inverse of A. Finally, we obtain the solution as a system of equations:

$f=\mathbf{A}^{-1} \cdot f^{*}=\mathbf{L} \cdot f^{*}$.

\subsection{Enhanced inversion}

Under specific atmospheric conditions, particles outside of the measurement range of the mobility particle size spectrometer might influence the results of the multiple-charge 
inversion. One example is the presence of significant numbers of coarse particles above the uppermost diameter channel of the mobility particle size spectrometer. The reason is that multiply charged particles of that population will appear as particle counts that are superimposed onto the signal of particles with low charge (single and double) in the nominal mobility particle size spectrometer range.

Disturbances could only completely be avoided if a mobility spectrometer were able to measure the PNSD across the entire range until the concentration reaches zero at the upper end. Due to technical reasons, however, the range of a mobility particle size spectrometer cannot be extended far beyond $1-2 \mu \mathrm{m}$. In practice, the range of common mobility particle size spectrometers employed in atmospheric measurements terminates between 500 and $900 \mathrm{~nm}$.

One approach has been to extrapolate the measured electrical particle mobility distribution into larger diameters (Brunner, 2007). This might be appropriate in the case of a continuously decreasing number concentration towards larger particles, but usually not when a significant coarse particle mode is present. Therefore, information on larger particles is needed, for example, using an aerodynamic particle sizer (APS) or an optical particle counter (OPC). Schladitz et al. (2009) first proposed the approach of correcting the measured EPMD for the effect of multiple charges prior to the actual inversion. We now present an implementation of this idea into the present inversion algorithm.

The real PNSD can be assumed to be a composition of a PNSD $f^{m}$ with $N$ sampling points, measured with a mobility particle size spectrometer, and an additional PNSD (volumeequivalent diameter) $f^{a}$ (in the following referred to as aPNSD), e.g. measured with an optical or aerodynamic particle size spectrometer, with $M$ sampling points.

$$
\boldsymbol{f}=\left(\begin{array}{c}
f_{1}^{m} \\
\vdots \\
f_{N}^{m} \\
f_{N+1}^{a} \\
\vdots \\
f_{N+M}^{a}
\end{array}\right) .
$$

The additional indices $m$ and $a$ are added to indicate that the sampling points belong to the mobility particle size spectrometer or the aPNSD. In accordance with Eq. (14) one can calculate the measured EPMD for the first $N$ sampling points, while the results for the aPNSD should be untouched by this algorithm:

$f_{i}^{*}= \begin{cases}\sum_{n=1}^{\infty} E\left(Z_{i}, n\right) f\left(\frac{1}{n} Z_{i}\right) & i \leq N \\ f\left(Z_{i}\right) & i>N .\end{cases}$

In case there is an overlap of the sampling points of both PNSDs, the function of the combined PNSD can be written

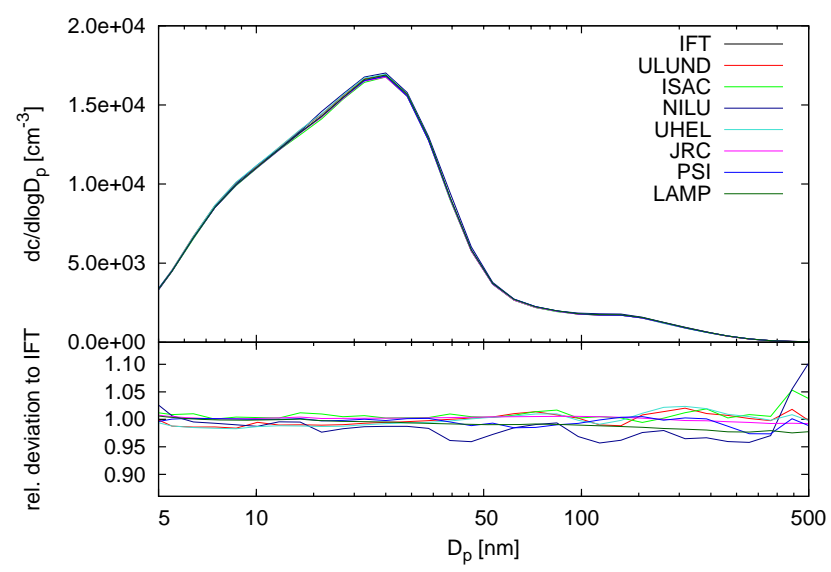

Fig. 2. Comparison of the performance of eight different multiplecharge inversion routines. Shown are the different resulting PNSDs based on the very same EPMD and their relative deviation to the new inversion routine. Reproduced from Wiedensohler et al. (2012) (IFT - Leibniz Institute for Tropospheric Research, Leipzig, Germany; ULUND - Lund University, Lund, Sweden; ISAC - Institute of Atmospheric Sciences and Climate, Bologna, Italy; NILU - Norwegian Institute for Air Research, Kjeller, Norway; UHEL - University of Helsinki, Helsinki, Finland; JRC - Joint Research Centre, Ispra, Italy; PSI - Paul Scherrer Institute, Villigen, Switzerland; LAMP - Laboratoire de Météorologie Physique, Clermont-Ferrand, France.

as

$$
\begin{aligned}
& f\left(\frac{1}{n} Z_{i}\right)= \\
& \left\{\begin{array}{ll}
\frac{1}{n} Z_{i}-Z_{j}^{m} \\
Z_{j+1}^{m}-Z_{j}^{m} \\
\frac{1}{n} Z_{i+1}-Z_{j}^{a} \\
\frac{Z_{j+1}^{a}-Z_{j}^{a}}{f_{j+1}^{a}} f_{j+1}^{a}+\frac{Z_{j+1}^{m} Z_{i}}{Z_{j+1}^{m}-Z_{j}^{m}} f_{j}^{m} Z_{j}^{a} & \frac{1}{n} Z_{i} \in\left(Z_{j+1}^{m}, Z_{j}^{m}\right) \wedge \frac{1}{n} Z_{i} \geq Z_{N}^{m} \\
Z_{j+1}^{a}-Z_{j}^{a} & \frac{1}{n} Z_{i} \in\left(Z_{j+1}^{a}, Z_{j}^{a}\right) \wedge \frac{1}{n} Z_{i}<Z_{N}^{m}
\end{array} .\right.
\end{aligned}
$$

In this case, the function values of the mobility particle size spectrometers are used as long as the multiply charged particles are in the detection range, or in other words, in the overlap range, we use the data of mobility particle spectrometer. $^{2}$ It is important to assign it to the one or the other PNSD or a weighted value between both. If it is assigned completely to both, it would be overvalued and considered wrongly twice. This overlap is useful as an indicator. If the enhanced algorithm and the measurements are correct, the inverted PNSD of the mobility particle spectrometer and the aPNSD of the optical or aerodynamic particle size spectrometer fit together.

\footnotetext{
${ }^{2}$ Using the aPNSD in the overlap range is also possible, as well as a weighted value between both
} 


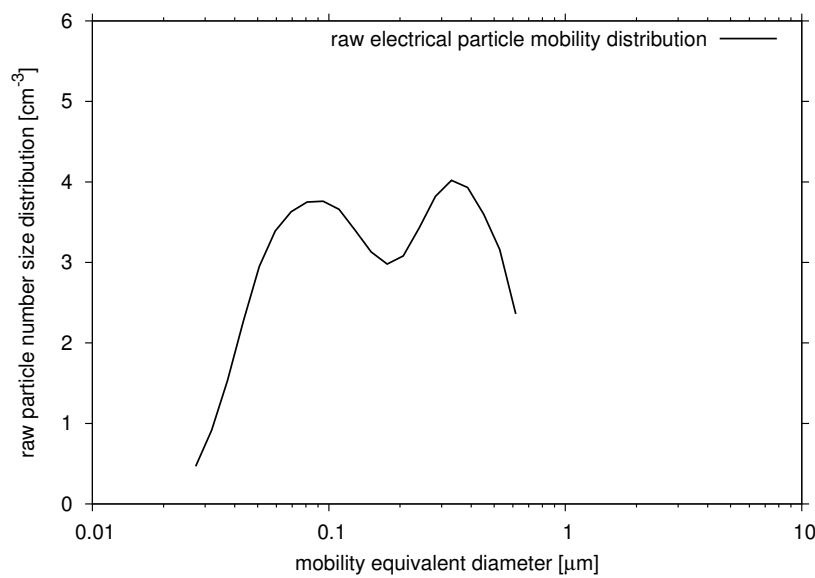

Fig. 3a. Raw electrical particle mobility distribution (EPMD) in plain concentration.

The resulting matrix consists of four sub-parts:

$a_{i j}=\left\{\begin{array}{ll}a_{i j}^{\mathrm{I}} & i \leq N \wedge j \leq N \\ a_{i j}^{\mathrm{II}} & i \leq N \wedge j>N \\ a_{i j}^{\mathrm{II}} & i>N \wedge j \leq N \\ a_{i j}^{\mathrm{IV}} & i>N \wedge j>N\end{array}\right.$.

Under these conditions, there should be an overlap range and in this range the mobility particle size spectrometer data should be used; the first part $a_{i j}^{\mathrm{I}}$ is identical to Eq. (16), describing the interaction of the multiple charges of the PNSD with itself.

$a_{i j}^{\mathrm{I}}=\sum_{n=1}^{\infty} E\left(Z_{i}, n\right)\left\{\begin{array}{ll}\frac{\frac{1}{n} Z_{i}-Z_{j-1}^{m}}{Z_{j}^{m}-Z_{j-1}^{m}} & \frac{1}{n} Z_{i} \in\left(Z_{j}^{m}, Z_{j-1}^{m}\right) \\ 1 & \frac{1}{n} Z_{i}=Z_{j}^{m} \\ \frac{Z_{j+1}^{m}-\frac{1}{n} Z_{i}}{Z_{j+1}^{m}-Z_{j}^{m}} & \frac{1}{n} Z_{i} \in\left(Z_{j+1}^{m}, Z_{j}^{m}\right) \\ 0 & \text { otherwise }\end{array}\right.$.

The second part $a_{i j}^{\mathrm{II}}$ describes the interaction of multiply charged particles of the aPNSD and the PNSD measured with the mobility particle size spectrometer:

$$
\begin{aligned}
a_{i j}^{\mathrm{II}} & =\sum_{n=1}^{\infty} E\left(Z_{i}, n\right) \\
& \begin{cases}\frac{1}{n} Z_{i}^{a}-Z_{j-1} & \frac{1}{n} Z_{i} \in\left(Z_{j}^{a}, Z_{j-1}^{a}\right) \wedge \frac{1}{n} Z_{i}<Z_{N}^{m} \\
Z_{j}^{a}-Z_{j-1}^{a} & \frac{1}{n} Z_{i}=Z_{j}^{a} \wedge \frac{1}{n} Z_{i}<Z_{N}^{m} \\
\frac{Z_{j+1}^{a}-\frac{1}{n} Z_{i}}{Z_{j+1}^{a}-Z_{j}^{a}} & \frac{1}{n} Z_{i} \in\left(Z_{j+1}^{a}, Z_{j}^{a}\right) \wedge \frac{1}{n} Z_{i}<Z_{N}^{m} \\
0 & \text { otherwise }\end{cases}
\end{aligned}
$$

Generally, due to the side condition for the overlap size range, the first entries of $a_{i j}^{\mathrm{II}}$ are empty (see Fig. 5b).

The last two parts are added to complete the rank of the matrix. Because the PNSD of the mobility particle size spectrometer has no effect on the aPNSD, $a_{i j}^{\mathrm{III}}$ is a zero matrix:

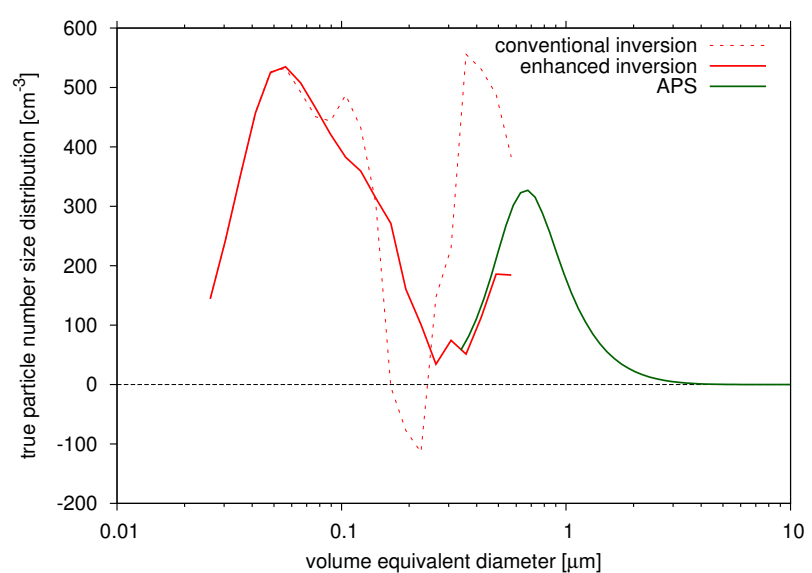

Fig. 3b. Results of the particle number size distribution (PNSD) obtained from multiple-charge inversion with different degrees of functionality. Solid green line: aerodynamic particle size distribution (APS). Red dashed line: multiple-charge inversion using only SMPS data. Red solid line: enhanced multiple-charge inversion combining APS and SMPS data.

$a_{i j}^{\mathrm{III}}=0 \quad \forall \quad i, j$.

More precisely, as already mentioned, the aPNSD should be untouched, so $a_{i j}^{\mathrm{IV}}$ is an identity matrix:

$a_{i j}^{\mathrm{IV}}=1 \quad \forall i=j$,

$\mathbf{A}=\left(\begin{array}{ccc|ccc}a_{11}^{\mathrm{I}} & \ldots & a_{1 N}^{\mathrm{I}} & a_{1(N+1)}^{\mathrm{II}} & \ldots & a_{1(N+M)}^{\mathrm{II}} \\ \vdots & \ddots & \vdots & \vdots & \ddots & \vdots \\ a_{N 1}^{\mathrm{I}} & \ldots & a_{N N}^{\mathrm{I}} & a_{N(N+1)}^{\mathrm{II}} & \ldots & a_{N(N+M)}^{\mathrm{II}} \\ \hline a_{(N+1) 1}^{\mathrm{II}} & \ldots & a_{(N+1) N}^{\mathrm{II}} & a_{(N+1)(N+1)}^{\mathrm{II}} & \ldots & a_{(N+1)(N+M)}^{\mathrm{IV}} \\ \vdots & \ddots & \vdots & \vdots & \ddots & \vdots \\ a_{(N+M) 1}^{\mathrm{II}} & \ldots & a_{(N+M) N}^{\mathrm{III}} & a_{(N+M)(N+1)}^{\mathrm{IV}} & \ldots & a_{(N+M)(N+M)}^{\mathrm{IV}}\end{array}\right)$.

By inserting the conditions to obtain the full rank, the matrix is

$$
\mathbf{A}=\left(\begin{array}{ccc|ccc}
a_{11}^{\mathrm{I}} & \ldots & a_{1 N}^{\mathrm{I}} & a_{1(N+1)}^{\mathrm{II}} & \ldots & a_{1(N+M)}^{\mathrm{II}} \\
\vdots & \ddots & \vdots & \vdots & \ddots & \vdots \\
0 & \ldots & a_{N N}^{\mathrm{I}} & a_{N(N+1)}^{\mathrm{II}} & \ldots & a_{N(N+M)}^{\mathrm{II}} \\
\hline 0 & \ldots & 0 & 1 & \ldots & 0 \\
\vdots & \ddots & \vdots & \vdots & \ddots & \vdots \\
0 & \ldots & 0 & 0 & \ldots & 1
\end{array}\right) .
$$

As the enhanced multiple-charge matrix is a sparsely occupied upper triangular matrix, we are able to solve

$f=\mathbf{L} \cdot f^{*}$.

However, the solution for the inverted PNSD is located in the first $N$ entries of the vector $\boldsymbol{f}$. 


\subsection{Error propagation}

An important thing to investigate is the error propagation by using an inversion algorithm. As an assumption, let the measured EPMD be influenced by an error due to counting statistics of the condensation particle counter (CPC). Thus the values of the measured and inverted distributions can be interpreted as random variables. The expectation value of a linear transformation $Y$ of a random variable $X$ is given by:

$E(Y)=E(a X+b)=a E(X)+b$.

Additionally, the sum of $n$ random variables $X_{i}$ is given by:

$E\left(\sum_{i=0}^{n} X_{i}\right)=\sum_{i=0}^{n} E\left(X_{i}\right)$.

As a result for the expectation value of the inverted PNSD we obtain

$E(\boldsymbol{f})=\mathbf{L} \cdot E\left(\boldsymbol{f}^{*}\right)$.

The variance of the linear transformation is given by:

$\operatorname{Var}(Y)=\operatorname{Var}(a X+b)=a^{2} \operatorname{Var}(X)$.

In contrast to the expectation value, for the variance of the sum of $n$ random variables $X_{i}$, the condition that the random variables are pairwise uncorrelated is required, i.e. statistically independent. According to the Bienaymé formula, the variance is given by:

$\operatorname{Var}\left(\sum_{i=0}^{n} X_{i}\right)=\sum_{i=0}^{n} \operatorname{Var}\left(X_{i}\right)$

Finally, for the variance of the inverted PNSD we obtain

$\operatorname{Var}(\boldsymbol{f})=\mathbf{L}_{\mathrm{var}} \cdot \operatorname{Var}\left(\boldsymbol{f}^{*}\right)$,

where $\mathbf{L}_{\mathrm{var}}$ is a matrix occupied by the squared entries of $\mathbf{L}$.

$l_{i j}^{\mathrm{var}}=l_{i j}^{2} \quad \forall \quad 0 \geq i, j \geq N$

A similar expression exists for the resulting variance of the $i$ th sampling point, under the assumption that the random variables are correlated:

$\operatorname{Var}\left(f_{i}\right)=\sum_{j=1}^{N} l_{i j} \sum_{k=1}^{N} l_{i k} \operatorname{Cov}\left(f_{j}^{*}, f_{k}^{*}\right)$,

where $\operatorname{Cov}\left(f_{j}^{*}, f_{k}^{*}\right)$ is the covariance of the $j$ th and $k$ th sampling point of the EPMD.

These aspects are valid either for the conventional or the enhanced inversion.

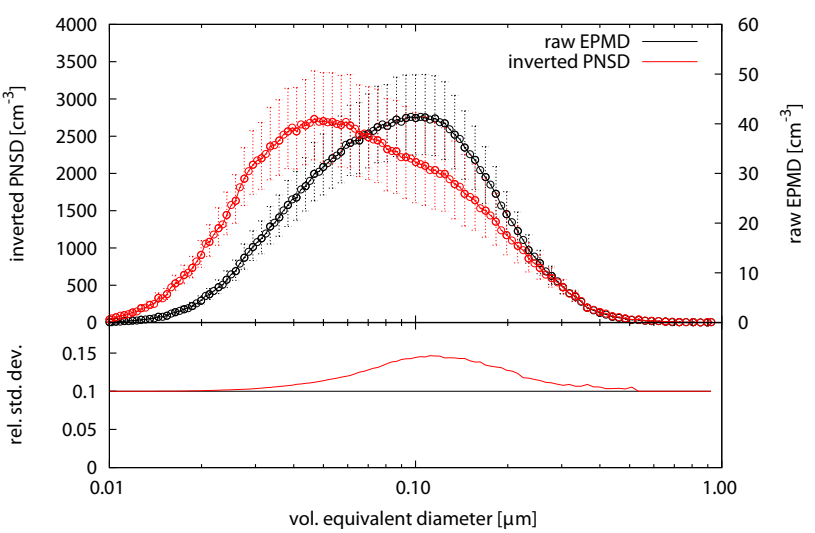

(a)

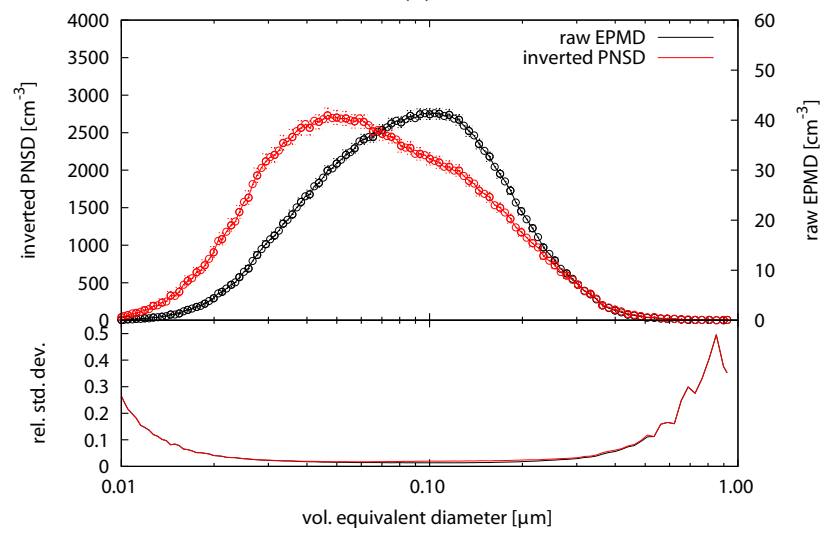

(b)

Fig. 4. Example of the error propagation of a mobility particle size spectrometer PNSD, Hohenpeissenberg, 23 February 2012, $1 \mathrm{~h}$ average 00:00-01:00 LT - EPMD (black) in plain concentration (secondary $y$ axis) and PNSD (red). (a) The fictive relative error of the results increases for sampling points influenced by multiple-charge correction from 10 to $15 \%$. (b) The relative error of the result is not significantly different from the input in the case of Poisson counting statistics.

\section{Results}

We devised a system of equations that directly transforms the measured EPMD to the real PNSD. This means an improvement in many respects.

1. The presented method preserves the original size bins of the measured electrical particle mobility distribution. This avoids unnecessary interpolations of the data into new size bins and the typically associated changes in the originally measured information.

2. The operator matrix and its inverted matrix are based on linear equations. With matrix inversion accomplished, the multiple-charge inversion problem reduces to a simple matrix multiplication, which is computationally very efficient if multiple EPMDs with the same mobility bins should be processed. 


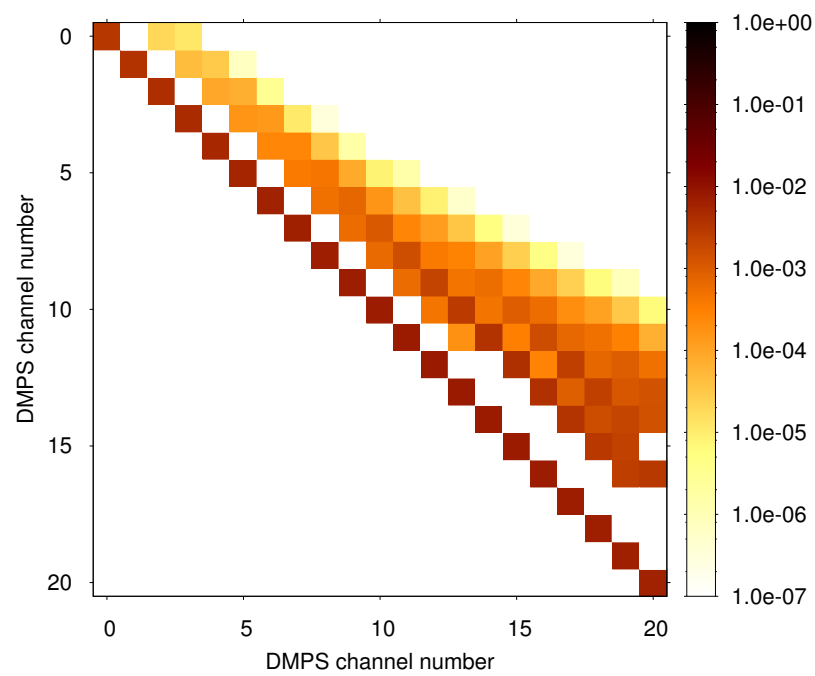

Fig. 5a. Visualization of the multiple-charge matrix using only mobility particle size spectrometer data.

3. The algorithm can easily incorporate information from additional sensors measuring particles outside of the nominal measurement range of the particle mobility size spectrometer.

4. Because the inversion reduces to linear matrix multiplication, computation of the effects of error propagation is straightforward (see Sect. 2.5).

5. The algorithm can handle all procedures using a constant or size-dependent aerodynamic shape factor.

\subsection{Validation of sub-micrometre EPMD inversion}

We compared this inversion routine with many other inversion routines within the framework of technical harmonization of particle mobility size spectrometry (Wiedensohler et al., 2012). Figure 2 visualizes the bias of our inversion routine against seven other contemporary inversion routines on the basis of an inversion of the same sub-micrometre EPMD. It can be seen that the different methods agree within a relative deviation of $5 \%$ for a wide particle size range. Larger deviations were explained by differing interpolation methods.

\subsection{Inversion of a wide size distribution combining SMPS and APS data}

As already mentioned, particles outside of the measurement range of the mobility particle size spectrometer might affect the results of the sub-micrometre multiple-charge inversion. In Fig. 3a, we illustrate the benefits of a multiple-charge inversion combining information from multiple sizing instruments, involving SMPS and APS, for the case of an atmospheric dust storm event in Morocco (Schladitz et al., 2009).

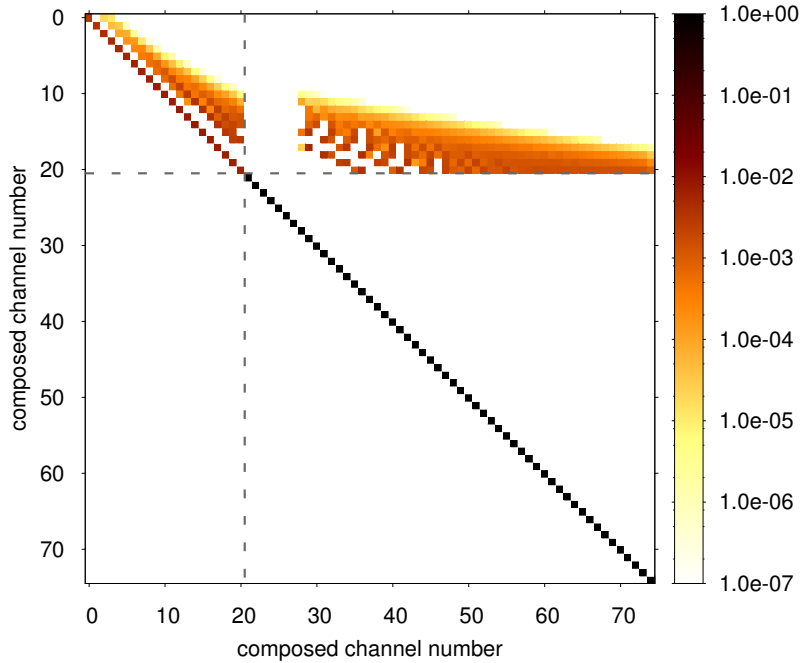

Fig. 5b. Visualization of the multiple-charge matrix for the enhanced case. The first quadrant corresponds to the matrix entries shown in Fig. 5a. The second quadrant describes the charge correction of the EPMD of the mobility particle size spectrometer with information from additional sensors such as an APS or OPC. The gap for the first entries of the second quadrant is due to the finite overlap of both sizing sensors.

Figure $3 \mathrm{~b}$ shows a bimodal shape of the EPMD, with number concentration maxima around 80 and $300 \mathrm{~nm}$. It is worth noting that in the uppermost SMPS sampling channel (corresponding to singly charged particles of $570 \mathrm{~nm}$ ), the EPMD drops to just half of the maximum value of the EPMD. At the same time, the APS size distributions reveals the presence of a significant coarse particle mode with a volume-equivalent modal diameter around $700 \mathrm{~nm}$. A multiple-charge inversion restricted to SMPS data will only necessarily need to interpret particle counts in the uppermost SMPS channel as singly charged particles. As can be seen in Fig. 3a, such an inversion causes an artificial depression in the final size distribution around $50-150 \mathrm{~nm}$ as a result of applying the multiple-charge inversion matrix. Also, the particle number size distribution at the upper tail of the distribution is heavily overestimated.

The quantitative consideration of the available APS data reveals that the particle counts in the uppermost SMPS channels in fact contain numerous multiple charges of particles beyond the SMPS measurement range. As can be seen in Fig. 3a, the enhanced inversion algorithm combining the information of both sensors successfully accounts for this effect, and yields a bimodal particle number size distribution involving a fine and a coarse particle mode that is both rather continuous and physically plausible. The particular result of the enhanced inversion algorithm depends on the specific assumptions regarding the aerodynamic particle shape factor and particle density. For the case shown in Fig. 3 we employed a coarse particle shape factor of 1.1 and a gravimetric density of $2.8 \mathrm{~g} \mathrm{~cm}^{-3}$, which are realistic values for mineral 
dust based on the results of previous field studies (Schladitz et al., 2009).

\subsection{Error propagation}

The propagation of possible measurement errors from the EPMD into the final PNSD is illustrated in Fig. 4a and 4b. The basis is a $1 \mathrm{~h}$ average of ambient EPMD at the rural observation site Hohenpeissenberg, Germany.

In Fig. 4a, we assume a fictive relative uncertainty (standard deviation) of $10 \%$ of particle number concentration measured in each channel of the EPMD. The error bars show the $95 \%$ confidence interval under the assumption of a lognormal-distributed random variable. Particles smaller than $20 \mathrm{~nm}$ are only influenced by singly charged particles; therefore the relative standard deviation is identical to the measured data. In the range from 20 to $600 \mathrm{~nm}$ an increase of the error of the inverted PNSD is noticeable. In this case, the error cumulates up to $15 \%$. Sampling points larger than $600 \mathrm{~nm}$ are also influenced by multiple charges, and one would expect also an increase of the error, but these multiply charged particles are outside of the detection range of the mobility particle size spectrometer. From this it follows that the relative standard deviation is identical to the measured values.

The same effect occurs when analysing the Poisson statistics based on experimental particle number counts for each raw concentration channel (see Fig. 4b), but not as significantly. The underlying reason is that the size range, influenced by multiply charged particles, exhibits the smallest errors, $1.3 \%$ at minimum.

The idea of the analytical error propagation is expandable in the case of correlated sampling points. Errors or uncertainties for the diameters or the $x$ values of the sampling points will influence the matrix elements. Therefore, it cannot be analysed in the same way. To investigate this effect, Monte Carlo simulations must be used further on.

\subsection{Suggested improvements and extensions}

Although the given algorithm represents a flexible tool, we see room for future improvements. A first issue would be the use of a uniform theory for the charging probability across the complete particle size range, especially for the enhanced inversion. A candidate would be the theory of Fuchs (1963).

The interpolations leading to Eq. (15) (Sect. 2.3) could be achieved by higher polynomial interpolation methods, possibly spline interpolation, rather than by linear interpolation.

However, it must be noted that these methods have their own disadvantages, as they need proper initial and boundary conditions and might also lead to artificial overshooting and therefore to an amplification of noise. For a dense grid of sampling points the gain of accuracy does not seem to be in proportion to the numerical effort.
An issue that is in principle relevant is the true deconvolution of the DMA transfer function, whose finite width is ignored by the present version of the algorithm. Moreover, the width, area and shape of the DMA transfer function usually depend on particle size, especially for the highly diffusive particles in the lower range of mobility particle size spectrometers (Flagan, 1999). It is possible to calculate the analytic solution of convolution, or the integral, of a triangular or bell-shaped transfer function, especially under the assumption of a linearly interpolated PNSD, and then determine the occupation of the matrix. Implementing such features would make this algorithm usable for very narrow PNSD and possibly improve the results for the smaller diameter range. It needs to be mentioned, however, that the transfer matrix would become a band matrix with entries on both sides on the main diagonal. The solution of such an inversion is more sensitive to variations in the input data and leads to an amplification of experimental noise. To avoid such a behaviour, numerically more demanding algorithms using smoothing constrains would be required (e.g. Talukdar and Swihart, 2003). The actual advantages of our algorithm - minimum resource consumption and high computational speed at an acceptable accuracy for atmospheric measurements including error propagation calculations - would be diminished.

\section{Conclusions}

We have presented the mathematical description of a multiple-charge inversion algorithm for mobility particle size spectrometers which is based on a forward transformation of the particle number size distribution (PNSD). The algorithm is based on very few approximations and interpolations, suggesting a number of advantages. Avoiding the interpolation of electrical particle mobility distributions (EPMD) onto a new grid helps to conserve the original experimental information. Due to the strict linear nature of the system of equations, the algorithm is extremely fast. Importantly, we encountered no serious deviations to previous inversion routines due to these simplifications.

Furthermore, the algorithm is able to balance a signal caused by multiply charged particles outside the nominal measurement range of a mobility particle size spectrometers by using additional information collected with optical or aerodynamic particle size spectrometers. This extended functionality was shown to be particularly relevant in atmospheres with numerous coarse particles, which could be resuspended mineral dust or sea spray particles.

Finally, because of this strict linear dependency, it is possible to calculate the error propagation, due to, for example, instrumental uncertainty or counting statistics in the inverted particle number size distribution. This is a frequently ignored aspect, but appears necessary to give the final particle number size distribution a statistical confidence and precision. 
All aspects of the algorithm are applicable for nonspherical particles, with non-sphericity being handled through an aerodynamic particle shape factor that transforms mobility equivalent into volume-equivalent diameters. This issue is particularly relevant for the enhanced inversion using information on super-micrometre dust particles.

\section{Appendix A}

\section{Conversion of number size distribution}

Transformation of $\mathrm{d} n / \mathrm{d} \ln Z$ to $\mathrm{d} n / \mathrm{d} \log D\left(\mathrm{~d} n / \mathrm{d} \log D_{\text {pve }}\right)$ :

$\frac{\mathrm{d} n}{\mathrm{~d} \log D_{\mathrm{pve}}}=\ln (10) \frac{D_{\mathrm{pve}}}{Z}\left|\frac{\mathrm{d} Z}{\mathrm{~d} D_{\mathrm{pve}}}\right| \frac{\mathrm{d} n}{\mathrm{~d} \ln Z}$,

where

$Z=\frac{n q_{\mathrm{e}}}{3 \pi \eta} \frac{C_{c}\left(D_{\mathrm{pve}}\right)}{\chi\left(D_{\mathrm{pve}}\right) D_{\mathrm{pve}}}$.

Let $C$ be the conversion factor for the density transformation:

$C\left(D_{\text {pve }}\right)=\ln (10) \frac{D_{\text {pve }}}{Z}\left|\frac{d Z}{d D_{\text {pve }}}\right|$,

or the inverse $C_{i}\left(D_{\mathrm{pve}}\right)=C\left(D_{\mathrm{pve}}\right)^{-1}$, and as a result

$\frac{\mathrm{d} n}{\mathrm{~d} \ln Z}=C_{i}\left(D_{\mathrm{pve}}\right) \frac{\mathrm{d} n}{\mathrm{~d} \log D_{\mathrm{pve}}}$.

\section{Appendix B}

\section{Linear interpolation}

The unknown real PNSD, the solution, is a continuous function; therefore we assume a linear interpolation for the given number of sampling points $N$.

$$
f(x)= \begin{cases}f_{1}^{\prime}\left(x-x_{1}\right)+f_{1} & x_{1} \leq x \leq x_{2} \\ \vdots & \vdots \\ f_{N-1}^{\prime}\left(x-x_{N-1}\right)+f_{N-1} & x_{N-1} \leq x \leq x_{N} \\ 0 & \text { otherwise }\end{cases}
$$

with

$f_{i}=f\left(x_{i}\right), \quad f_{i}^{\prime}=\frac{f_{i+1}-f_{i}}{x_{i+1}-x_{i}}$.

For $x \in\left(x_{i}, x_{i+1}\right)$ we obtain the function value

$$
f(x)=\frac{x-x_{i}}{x_{i+1}-x_{i}} f_{i+1}+\frac{x_{i+1}-x}{x_{i+1}-x_{i}} f_{i} .
$$

\section{Appendix C}

\section{Multiple-charge inversion with CCNC}

Using a CPC, the EPMD is given by:

$$
f_{i}^{*}=f^{*}\left(Z_{i}\right)=\sum_{n=1}^{\infty} E\left(Z_{i}, n\right) f\left(\frac{1}{n} Z_{i}\right) .
$$

Using a CCNC instead of a CPC modifies the efficiency. In addition, we need the size-dependent activation $a(D)$ :

$$
f_{C i}^{*}=f^{*}\left(Z_{i}\right)=\sum_{n=1}^{\infty} E\left(Z_{i}, n\right) a\left(\frac{1}{n} Z_{i}\right) f\left(\frac{1}{n} Z_{i}\right) .
$$

With $a\left(\frac{1}{n} Z_{i}\right) f\left(\frac{1}{n} Z_{i}\right)=f_{C}\left(\frac{1}{n} Z_{i}\right)$, we obtain

$f_{C}^{*}=\mathbf{A} \cdot f_{C}$,

where $\boldsymbol{f}_{C}$ is the real PNSD of the activated particles and $f_{C}^{*}$ is the measured EPMD when using a CCNC instead of a CPC.

Acknowledgements. We would like to thank J.-L. Jimenez for editing, as well as the anonymous referees for their critical feedbacks.

Edited by: J.-L. Jimenez

\section{References}

Alofs, D. J. and Balakumar, P.: Inversion to obtain aerosol size distributions from measurements with a differential mobility analyzer, J. Aerosol Sci., 13, 513-527, 1982.

Birmili, W., Stratmann, F., Wiedensohler, A., Covert, D., Russel, L. M., and Berg, O.: Determination of differential mobility analyzer transfer functions using identical instruments in series, Aerosol Sci. Technol., 27, 215-223, 1997.

Birmili, W., Schepanski, K., Ansmann, A., Spindler, G., Tegen, I., Wehner, B., Nowak, A., Reimer, E., Mattis, I., Müller, K., Brüggemann, E., Gnauk, T., Herrmann, H., Wiedensohler, A., Althausen, D., Schladitz, A., Tuch, T., and Löschau, G.: A case of extreme particulate matter concentrations over Central Europe caused by dust emitted over the southern Ukraine, Atmos. Chem. Phys., 8, 997-1016, doi:10.5194/acp-8-997-2008, 2008.

Brunner, J.: Particle measurements (size distribution and number) with a SMPS - Technical basis and evaluation procedures (translated from "Partikelmessungen (Grössenverteilung und Anzahl) mit einem SMPS - Grundlagen und Auswerteverfahren"), Tech. Rep. No. 20051105, Stadt Zürich, Umwelt- und Gesundheitsschutz Zürich UGZ, 8021 Zürich, Revision 2.1, 2007.

Fiebig, M., Stein, C., Schröder, F., Feldpausch, P., and Petzold, A.: Inversion of data containing information on the aerosol particle size distribution using multiple instruments, J. Aerosol Sci., 36, 1353-1372, 2005.

Flagan, R. C.: On differential mobility analyzer resolution, Aerosol Sci. Technol., 30, 556-570, 1999. 
Fuchs, N. A.: On the stationary charge distribution on aerosol Particles in a bipolar ionic atmosphere, Pure Appl. Geophys., 56, 185-193, 1963.

Gunn, R.: The statistical electrification of aerosols by ionic diffusion, J. Colloid Sci., 10, 107-119, 1955.

Hinds, W. C.: Aerosol Technology: Properties, Behavior, and Measurement of Airborne Particles, Wiley-Interscience, 2nd Edn., 1999.

Kandlikar, M. and Ramachandran, G.: Inverse methods for analysing aerosol spectrometer measurements: a critical review, J. Aerosol Sci., 30, 413-437, 1999.

Knutson, E. O. and Whitby, K. T.: Aerosol classification by electrical mobility apperatus, theory, and applications, J. Aerosol Sci., 6, 443-451, 1975.

Kousaka, Y., Okuyama, K., and Adachi, M.: Determination of particle size distribution of ultra-fine aerosols using a differential mobility analyzer, Aerosol Sci. Technol., 4, 209-225, 1985.

Ku, B. K., Deye, G. J., Kulkarni, P., and Baron, P. A.: Bipolar diffusion charging of high-aspect ratio aerosols, J. Electrostatics, 69, 541-647, 2011.

McMurry, P. H.: A review of atmospheric aerosol measurements, Atmos. Environ., 34, 1959-1999, 2000.

Rogak, S. N. and Flagan, R. C.: Bipolar diffusion charging of spheres and agglomarated aerosol particles, J. Aerosol Sci., 23, 693-710, 1992.

Schladitz, A., Müller, T., Kaaden, N., Maasling, A., Kandler, K., Ebert, M., Weinbruch, S., Deutscher, C., and Wiedensohler, A.: In situ measurements of optical properties at Tinfou (Morocco) during the Saharan Mineral Dust Experiment SAMUM 2006, Tellus, 61B, 64-78, 2009.
Stratmann, F., Kauffeldt, T., Hummes, D., and Fissan, H.: Differential electrical mobility analysis: A theoretical study, Aerosol Sci. Technol., 26, 368-383, 1997.

Talukdar, S. S. and Swihart, M. T.: An improved data inversion program for obtaining aerosol size distributions from scanning differential mobility analyzer data, Aerosol Sci. Technol., 37, 145161, 2003.

Wiedensohler, A.: An approximation of the bipolar charge distribution for particles in the submicron size range, J. Aerosol Sci., 19, 387-389, 1988.

Wiedensohler, A., Birmili, W., Nowak, A., Sonntag, A., Weinhold, K., Merkel, M., Wehner, B., Tuch, T., Pfeifer, S., Fiebig, M., Fjäraa, A. M., Asmi, E., Sellegri, K., Depuy, R., Venzac, H., Villani, P., Laj, P., Aalto, P., Ogren, J. A., Swietlicki, E., Williams, P., Roldin, P., Quincey, P., Hüglin, C., Fierz-Schmidhauser, R., Gysel, M., Weingartner, E., Riccobono, F., Santos, S., Grüning, C., Faloon, K., Beddows, D., Harrison, R., Monahan, C., Jennings, S. G., O’Dowd, C. D., Marinoni, A., Horn, H.-G., Keck, L., Jiang, J., Scheckman, J., McMurry, P. H., Deng, Z., Zhao, C. S., Moerman, M., Henzing, B., de Leeuw, G., Löschau, G., and Bastian, S.: Mobility particle size spectrometers: harmonization of technical standards and data structure to facilitate high quality long-term observations of atmospheric particle number size distributions, Atmos. Meas. Tech., 5, 657-685, doi:10.5194/amt5-657-2012, 2012. 